\title{
IS THE UV ALIGNMENT EFFECT PRESENT IN LOW REDSHIFT RADIO GALAXIES ?
}

\author{
ANDREA CIMATTI AND SPERELLO DI SEREGO ALIGHIERI \\ Osservatorio Astrofisico di Arcetri \\ Largo E. Fermi 5, I-50125, Firenze, Italy
}

\section{Introduction}

When a FRII radio galaxy at $z>0.7$ is observed in the optical, its continuum appears extended and aligned with the radio axis. This phenomenon occurs actually when the optical bands start to sample the rest-frame UV, and it was called alignment effect (McCarthy et al. 1987). The UV continuum of high $z$ radio galaxies shows also strong linear polarization due to scattering of anisotropic radiation escaping from the obscured quasar nucleus (di Serego Alighieri, Cimatti \& Fosbury 1994). However, the observations of the UV continuum have been concentrated mostly on high $z$ radio galaxies, leaving open a main question : is the alignment effect an evolutionary phenomenon present only at high $z$, or is it simply a selection effect due to the K-correction ? In order to investigate the origin and evolution of the UV alignment effect, we have started a ground-based imaging and polarimetric survey for studying the UV continuum in FR II radio galaxies with $0.1<z<0.5$. Depending on the redshift, the filters $U$ and $B$ can be used to sample the UV continuum free from strong emission lines, and in the same rest-frame spectral region observed in radio galaxies at higher redshift. The survey is in progress and here we present only our first results.

\section{Results and first implications}

We observed 7 galaxies and detected extended and polarized UV continua in 3 of them. Although the sample is small and incomplete, some implications can already be drawn. $\bullet$ Powerful radio galaxies with $0.1 \leq z<0.5 d o$ not always show the UV alignment effect. We recall that the UV continuum 
of powerful radio galaxies at higher redshift is always aligned with the radio axis. - This results implies that the alignment effect is not simply due to a K-correction effect. - The round UV continua tend to show lower polarization. In comparison, the aligned UV continua are highly polarized with the electric vector perpendicular to the axis defined by the UV continuum morphology. - As in high redshift radio galaxies, the dominant polarization mechanism is scattering by dust and/or electrons of the anisotropic radiation emitted by an obscured quasar nucleus. In fact, the UV-optical continua can be successfully fitted with scattered quasar radiation + stellar light. - In this respect, powerful radio galaxies which show the alignment effect mimic the properties observed at high redshift. The most striking example is provided by 3C 195 (Cimatti \& di Serego Alighieri 1995).

\section{What triggers the alignment effect ?}

Because in low $z$ powerful radio galaxies the alignment effect can be absent, we can conclude that it is an evolutionary effect, meaning that it is always occurring at $z>0.7$, but only sometimes at lower $z$. This evolution can be related to the increase of the radio power at high redshift (Dunlop \& Peacock 1990), and/or the evolution of the environment of the radio galaxies (ISM and/or merging companion galaxies). Recent results have suggested that the alignment effect at $z \sim 1$ depends on the radio power (Dunlop \& Peacock 1993), but more observations at different redshifts are still required. Concerning the degree of polarization, we note that in case of scattering, $P$ depends on the geometrical configuration, on the nature and size of the scattering particles and, in presence of unpolarized radiation, on the luminosity of incident radiation beam. The unified model of powerful radioloud AGN predicts that anisotropic radiation must be present in all FR II radio galaxies with narrow-lined optical spectra, irrespectively of the redshift. However, we do not observe strong scattered polarized light in all the narrow-lined radio galaxies at low redshift. The origin of this 'evolution' of the UV polarization will be investigated in detail by using our coming sample of low redshift radio galaxies.

\section{References}

Cimatti, A., di Serego Alighieri, S., 1995, MNRAS, 273, L7

di Serego Alighieri, S., Cimatti, A., Fosbury, R.A.E., 1994, ApJ, 431, 123

Dunlop, J.S., Peacock, J., 1990, MNRAS, 247, 19

Dunlop, J.S., Peacock, J., 1993, MNRAS, 263, 936

McCarthy, P.J., van Breugel, W.J.M., Spinrad, H., Djorgovski, S., 1987, ApJ, 321, L29 